\title{
Salvaging the supernatant: next generation cytopathology for solid tumor mutation profiling
}

\author{
Sinchita Roy-Chowdhuri ${ }^{1}$ - Meenakshi Mehrotra ${ }^{2}$ - Ana Maria Bolivar ${ }^{3} \cdot$ Rashmi Kanagal-Shamanna $^{2}$. \\ Bedia A. Barkoh ${ }^{2} \cdot$ Brette Hannigan $^{3} \cdot$ Stephanie Zalles $^{3} \cdot$ Wenrui Ye $^{3} \cdot$ Dzifa Duose $^{4} \cdot$ Russell Broaddus $^{1}$. \\ Gregg Staerkel $^{1} \cdot$ Ignacio Wistuba $^{4} \cdot$ L. Jeffrey Medeiros ${ }^{2} \cdot$ Rajyalakshmi Luthra $^{2,4}$
}

Received: 23 August 2017 / Revised: 27 November 2017 / Accepted: 3 December 2017 / Published online: 20 February 2018

(c) United States \& Canadian Academy of Pathology 2018

\begin{abstract}
With the expanding role of targeted therapy in patients with solid tumors, pathologists face the daunting task of having to maximize limited volume tissue obtained by fine needle aspiration for a variety of molecular tests. While most molecular studies on fine needle aspiration samples have been reported using cellular material, recent studies have shown that a substantial amount of DNA can be retrieved from the supernatant fluid of aspirate needle rinses after cell pelleting for cytospin or cell block preparations. In routine clinical workflow, the supernatant is discarded; however this fluid may provide a complementary source of DNA for tumor mutational profiling. In this study, we evaluated the post-centrifuged supernatant from 25 malignant and 10 benign fine needle aspiration needle rinses. The mean and median DNA yields from the supernatants were $445 \mathrm{ng}$ and $176.4 \mathrm{ng}$ (range, 15.1-2958 ng), respectively. Next generation sequencing using the Ion AmpliSeq Cancer Hotspot Panel v2 detected somatic mutations in all 25 malignant samples. No mutations were detected in any of the benign samples tested. When available, mutations detected in the supernatant fluid were compared to the next generation sequencing analysis performed on a prior or concurrent surgical specimen from the same patient and showed $100 \%$ concordance. In a subset of cases $(n=19)$ mutations in EGFR, KRAS, BRAF, PIK3CA, and NRAS were successfully confirmed by droplet digital PCR, providing an orthogonal platform for mutation analysis. In summary, in this study we show that post centrifuged supernatants from fine needle aspiration needle rinses can provide a robust substrate for expanded mutation profiling by next generation sequencing, as well as hotspot mutation testing by droplet digital PCR. The ability to detect somatic mutations from otherwise discarded supernatant fluids offers the ability to triage and effectively utilize limited volume fine needle aspiration samples when multiple molecular tests are requested, without the need to re-biopsy for additional tissue samples.
\end{abstract}

Sinchita Roy-Chowdhuri and Meenakshi Mehrotra contributed equally to this work.

Sinchita Roy-Chowdhuri

sroy2@mdanderson.org

1 Department of Pathology, Division of Pathology and Laboratory Medicine, The University of Texas MD Anderson Cancer Center, Houston, TX, USA

2 Department of Hematopathology, Division of Pathology and Laboratory Medicine, The University of Texas MD Anderson Cancer Center, Houston, TX, USA

3 Diagnostic Genetics, School of Health Professions, The University of Texas MD Anderson Cancer Center, Houston, TX, USA

4 Department of Translational Molecular Pathology, Division of Pathology and Laboratory Medicine, The University of Texas MD Anderson Cancer Center, Houston, TX, USA

\section{Introduction}

In an era of targeted therapy, there is a critical need to efficiently utilize limited volume tissue samples obtained through minimally invasive procedures for molecular testing. With the increasing numbers of molecular tests requested for solid organ malignancies, pathologists frequently face the daunting task of having to triage and maximize small samples for a variety of molecular tests, including mutation analysis, fluorescence in situ hybridization, and immunohistochemistry staining for prognostic and/or predictive biomarkers [1]. Although the method of tissue sampling, for example core needle biopsy vs. fine needle aspiration, frequently depends on the size and location of the lesion, the preference of the proceduralist, and institutional practice, a large fraction of patients with 
solid tumors are diagnosed by fine needle aspiration [2-4]. The constantly expanding list of clinically relevant biomarkers, therefore, underscores the need for highthroughput multiplexed molecular assays that can be performed on low-volume cytology samples.

The role of next generation sequencing, for multiplexed multigene mutation analysis, in cytology is well-established in the literature and many institutions utilize a variety of cytologic substrates including direct smears, cell block preparations, and liquid-based cytology for next generation sequencing [5-22]. However, due to the multitude of biomarkers requested, the limited volume tissue available on the cytology slides may not always be adequate. Recent studies have shown that substantial amounts of nucleic acid can be retrieved from the supernatant fluid of fine needle aspiration needle rinses after centrifugation and cell pelleting [23-27]. In routine clinical workflow, this supernatant fluid is typically discarded; however tumor DNA that may be left in this fluid can be effectively utilized for characterizing molecular changes in tumor.

Studies using supernatant fluid of pancreatic fine needle aspiration samples fixed in Saccomano's fixative, have shown that adequate and amplifiable DNA can be extracted for KRAS (codon 12 and 13) mutation analysis by pyrosequencing [23-26], even when the cellular content (smears) from the corresponding cytology sample lacked sufficient cellularity [24]. Another recent study using thyroid fine needle aspiration samples reported the detection of $B R A F$ mutations by pyrosequencing using supernatants from aspirates collected in CytoLyt [27]. Therefore, in situations where there is limited tissue for evaluation of molecular markers, judicious triaging of the cytology specimen with effective utilization of the entire sample, including both the cellular and the supernatant components, may be needed. While most of the published studies have focused on single gene analysis in pancreaticobiliary and thyroid tumors, to our knowledge, this is the first report on the feasibility of a multiplexed, multigene mutation analysis, with high analytical sensitivity, in a wide variety of tumor types using the discarded supernatant fluids. Here, we evaluate the utility of the supernatant fluid following centrifugation of fine needle aspiration samples of solid tumors, for the detection of clinically relevant mutations using next generation sequencing.

\section{Materials and Methods}

\section{Sample selection}

All image-guided fine needle aspirations performed at our institution include 2-3 passes using 20-25 gauge needles. Expelled tissue from these needle aspirates are processed as direct smears, Diff-Quik and/or Papanicolaou stained, while residual tissue in the needle is rinsed into $10 \mathrm{ml}$ of RPMI media [28]. The needle rinse is centrifuged at $1500 \mathrm{rpm}$ for $10 \mathrm{~min}$ and the centrifuged cellular material is processed either as a cytospin preparation or as a formalin-fixed paraffin-embedded cell block [5]. The supernatant fluid is typically discarded following centrifugation.

Following approval by the Institutional Review Board, supernatant fluids from fine needle aspiration needle rinses $(n=35)$ were collected after the diagnostic cytology report had been finalized and prior to discard. The tumor samples included, metastatic melanoma $(n=7)$, pancreatic adenocarcinoma $(n=4)$ with one metastatic to the liver $(n=1)$, lung adenocarcinoma $(n=3)$, poorly differentiated carcinoma of unknown primary site in liver and lymph node ( $n$ $=3$ ), colorectal adenocarcinoma metastatic to liver and lymph node $(n=2)$, small cell carcinoma in lung and metastatic to lymph node $(n=2)$, breast adenocarcinoma metastatic to liver $(n=1)$, urothelial carcinoma metastatic to lung $(n=1)$, and hepatocellular carcinoma $(n=1)$. Ten benign lymph nodes were also assessed as a negative control to assess assay specificity. All samples were collected from January 1, 2017 through April 2017. Only samples where supernatants were decanted and salvaged prior to addition of formalin for a cell block preparation were selected for the study. Consecutive cases with a malignant diagnosis of adenocarcinoma, carcinoma (poorly differentiated, small cell, hepatocellular, and urothelial), and melanoma were selected. The selection of the lung adenocarcinoma and metastatic melanoma cases were based on the availability of next generation sequencing mutation analysis of a concurrent or prior surgical specimen for comparison and the presence of a known mutation in a clinically relevant gene. The benign lymph node aspirates were selected consecutively based on availability of supernatants, as previously mentioned.

\section{Sample cellularity and molecular adequacy assessment}

The corresponding cytology smears and cytospin preparation and/or cell block sections from the malignant tumors were evaluated for adequacy for molecular testing by next generation sequencing. The adequacy criteria included a cell block with at least 300 cells per section and a minimum of $20 \%$ tumor cellularity, and/or 1 or 2 smears with at least 1000 cells and a minimum tumor fraction of $20 \%$ [2]. Cases that did not meet the adequacy criteria were rejected as "quantity not sufficient". Cases that met the adequacy criteria were assessed individually to evaluate the amount of tissue present on the slides and determine if the sample would qualify for next generation sequencing. Cases that were considered adequate by the outlined criteria but had 
Table 1 Mutational analysis of supernatant fluids from fine needle aspiration samples

\begin{tabular}{|c|c|c|c|c|c|c|c|c|c|}
\hline No. & Site & Diagnosis & $\begin{array}{l}\text { Total } \\
\text { DNA } \\
\text { (ng) }\end{array}$ & Gene & Mutation detected & $\begin{array}{l}\text { Supernatant } \\
\text { NGS AF\% }\end{array}$ & $\begin{array}{l}\text { Supernatant } \\
\text { ddPCR AF\% }\end{array}$ & $\begin{array}{l}\text { Tissue }(\mathrm{C} / \mathrm{P}) \\
\text { NGS AF\% }\end{array}$ & $\begin{array}{l}\text { Cytology } \\
\text { Adequacy }\end{array}$ \\
\hline 1 & Lung & $\mathrm{ADC}$ & 24.7 & $E G F R$ & p.L858R & 23.7 & 19 & $24.9^{\mathrm{c}}$ & QNS \\
\hline \multirow[t]{2}{*}{2} & \multirow[t]{2}{*}{ Lung } & \multirow[t]{2}{*}{$\mathrm{ADC}$} & \multirow[t]{2}{*}{180.6} & $E G F R$ & p.E746_A750delins & 54.7 & N.A. & $43.5^{c}$ & LV \\
\hline & & & & $E G F R$ & p.T790M & 47.5 & 48 & $37.9^{\mathrm{c}}$ & \\
\hline 3 & Lung & $\mathrm{ADC}$ & 312 & $K R A S$ & p.G12V & 9.6 & 16.7 & $24.1^{\mathrm{c}}$ & LV \\
\hline \multirow[t]{2}{*}{4} & \multirow[t]{2}{*}{ Liver } & \multirow[t]{2}{*}{ Met Mel } & \multirow[t]{2}{*}{1986} & $B R A F$ & p.V600E & 27.5 & 30.1 & $51.1^{\mathrm{c}}$ & $\mathrm{AD}$ \\
\hline & & & & TP53 & p.G266V & 26.5 & N.A. & $72.6^{c}$ & \\
\hline \multirow[t]{3}{*}{5} & \multirow{3}{*}{$\begin{array}{l}\text { Lymph } \\
\text { Node }\end{array}$} & \multirow[t]{3}{*}{ Met Mel } & \multirow[t]{3}{*}{507} & $N R A S$ & p.Q61R & 48 & 46.4 & $28.8^{p}$ & $\mathrm{AD}$ \\
\hline & & & & TP53 & p.G266V & 72 & N.A. & $15.4^{\mathrm{p}}$ & \\
\hline & & & & SMAD4 & p.L109R & 75.7 & N.A. & $15.5^{\mathrm{p}}$ & \\
\hline \multirow[t]{2}{*}{6} & \multirow[t]{2}{*}{ Soft tissue } & \multirow[t]{2}{*}{ Met Mel } & \multirow[t]{2}{*}{2958} & $N R A S$ & p.Q61K & 63 & 65.3 & $35.5^{\mathrm{p}}$ & $\mathrm{AD}$ \\
\hline & & & & $P I K 3 C A$ & p.E542K & 67.3 & 67.9 & N.A. ${ }^{a}$ & \\
\hline 7 & Chest wall & Met Mel & 229.2 & $N R A S$ & p.Q61R & 71.8 & 73.9 & $79.2^{\mathrm{c}}$ & $\mathrm{AD}$ \\
\hline 8 & Lung & Met Mel & 99.6 & $B R A F$ & p.V600E & 6.7 & 6.4 & $35.5^{\mathrm{c}}$ & QNS \\
\hline 9 & Soft tissue & Met Mel & 565.8 & $B R A F$ & p.V600E & 56.9 & 58.6 & $57.1^{\mathrm{p}}$ & $\mathrm{AD}$ \\
\hline 10 & Soft tissue & Met Mel & 176.4 & $N R A S$ & p.Q61R & 51.7 & 50.8 & $69^{p}$ & $\mathrm{AD}$ \\
\hline \multirow[t]{2}{*}{11} & \multirow[t]{2}{*}{ Liver } & \multirow[t]{2}{*}{ Met CRC } & \multirow[t]{2}{*}{726} & $K R A S$ & p.G12D & 17.5 & 19.6 & N.A. & QNS \\
\hline & & & & TP53 & p.R248Q & 11.4 & N.A. & N.A. & \\
\hline \multirow[t]{2}{*}{12} & \multirow[t]{2}{*}{ Pancreas } & $\mathrm{ADC}$ & 606 & $K R A S$ & p.Q61H & 47.1 & 46 & N.A. & LV \\
\hline & & & & SMAD4 & p.R361C & 47.6 & N.A. & N.A. & \\
\hline 13 & Pancreas & $\mathrm{ADC}$ & 124.8 & $K R A S$ & p.G12D & 16.3 & 15.2 & N.A. & QNS \\
\hline & & & & SMAD4 & p.R445* & 10 & N.A. & N.A. & \\
\hline 14 & Pancreas & $\mathrm{ADC}$ & 126.6 & $K R A S$ & p.G12D & 26.6 & 25.9 & N.A. & $\mathrm{AD}$ \\
\hline & & & & TP53 & p.R213* & 38.9 & N.A. & N.A. & \\
\hline 15 & Pancreas & $\mathrm{ADC}$ & 44.3 & $K R A S$ & p.G12D & 5.5 & 6.4 & N.A. & QNS \\
\hline & & & & SMAD4 & p.R361H & 5.4 & N.A. & N.A. & \\
\hline 16 & Liver & Met PanADC & 38.3 & $K R A S$ & p.G12V & 14.6 & 12.7 & N.A. & LV \\
\hline 17 & Lung & Met UroCa & 516 & $F G F R 3$ & p.S249C & 20.1 & N.A. & N.A. & $\mathrm{AD}$ \\
\hline 18 & Liver & $\mathrm{HCC}$ & 56 & TP53 & p.G245D & 70.3 & N.A. & N.A. & QNS \\
\hline 19 & Liver & Met BrADC & 1644 & TP53 & p.R273H & 83.9 & N.A. & N.A. & $\mathrm{AD}$ \\
\hline & & & & PTEN & p.S10I & 85.8 & N.A. & N.A. & \\
\hline 20 & Lymph & Met CRC & 139.2 & $R E T$ & p.A146T & 25.26 & N.A. & N.A. & LV \\
\hline & Node & & & TP53 & p.R273C & 24.1 & N.A. & N.A. & \\
\hline 21 & Liver & $\mathrm{PDCa}^{\mathrm{b}}$ & 882 & IDHI & p.R132C & 22.52 & N.A. & N.A. & $\mathrm{AD}$ \\
\hline & & & & TP53 & p.R231* & 30.56 & N.A. & N.A. & \\
\hline & & & & $P I K 3 C A$ & p.E542K & 9.35 & 9.3 & N.A. & \\
\hline 22 & $\begin{array}{l}\text { Lymph } \\
\text { Node }\end{array}$ & $\mathrm{PDCa}$ & 1536 & TP53 & p.R231* & 53.4 & N.A. & N.A. & LV \\
\hline 23 & Liver & $\mathrm{PDCa}^{\mathrm{b}}$ & 107.4 & KRAS & p.G12D & 12.9 & 11.6 & N.A. & QNS \\
\hline 24 & Lung & SCC & 233.4 & $A T M$ & p.F858L & 47.7 & N.A. & N.A. & $\mathrm{AD}$ \\
\hline & & & & TP53 & p.H168R & 90.7 & N.A. & N.A. & $\mathrm{AD}$ \\
\hline 25 & $\begin{array}{l}\text { Lymph } \\
\text { Node }\end{array}$ & SCC & 252.6 & TP53 & p.A276D & 86.9 & N.A. & N.A. & N.A. \\
\hline 26 & $\begin{array}{l}\text { Lymph } \\
\text { Node }\end{array}$ & No tumor & 102.6 & NMD & N.A. & N.A. & N.A. & N.A. & N.A. \\
\hline 27 & $\begin{array}{l}\text { Lymph } \\
\text { Node }\end{array}$ & No tumor & 128.4 & NMD & N.A. & N.A. & N.A. & N.A. & N.A. \\
\hline 28 & & No tumor & 49.8 & NMD & N.A. & N.A. & N.A. & N.A. & N.A. \\
\hline
\end{tabular}


Table 1 (continued)

\begin{tabular}{|c|c|c|c|c|c|c|c|c|c|}
\hline No. & Site & Diagnosis & $\begin{array}{l}\text { Total } \\
\text { DNA } \\
(\mathrm{ng})\end{array}$ & Gene & Mutation detected & $\begin{array}{l}\text { Supernatant } \\
\text { NGS AF\% }\end{array}$ & $\begin{array}{l}\text { Supernatant } \\
\text { ddPCR AF\% }\end{array}$ & $\begin{array}{l}\text { Tissue }(\mathrm{C} / \mathrm{P}) \\
\text { NGS AF\% }\end{array}$ & $\begin{array}{l}\text { Cytology } \\
\text { Adequacy }\end{array}$ \\
\hline 29 & $\begin{array}{l}\text { Lymph } \\
\text { Node } \\
\text { Lymph } \\
\text { Node }\end{array}$ & No tumor & 220.8 & NMD & N.A. & N.A. & N.A. & N.A. & N.A. \\
\hline 30 & $\begin{array}{l}\text { Lymph } \\
\text { Node }\end{array}$ & No tumor & 141 & NMD & N.A. & N.A. & N.A. & N.A. & N.A. \\
\hline 31 & $\begin{array}{l}\text { Lymph } \\
\text { Node }\end{array}$ & No tumor & 660 & NMD & N.A. & N.A. & N.A. & N.A. & N.A. \\
\hline 32 & $\begin{array}{l}\text { Lymph } \\
\text { Node }\end{array}$ & No tumor & 122.4 & NMD & N.A. & N.A. & N.A. & N.A. & N.A. \\
\hline 33 & $\begin{array}{l}\text { Lymph } \\
\text { Node }\end{array}$ & No tumor & 43.2 & NMD & N.A. & N.A. & N.A. & N.A. & N.A. \\
\hline 34 & $\begin{array}{l}\text { Lymph } \\
\text { Node }\end{array}$ & No tumor & 21.2 & NMD & N.A. & N.A. & N.A. & N.A. & N.A. \\
\hline 35 & $\begin{array}{l}\text { Lymph } \\
\text { Node }\end{array}$ & No tumor & 15.1 & NMD & N.A. & N.A. & N.A. & N.A. & N.A. \\
\hline
\end{tabular}

c: Mutation detected and confirmed on concurrent surgical specimen (also shown for each surgical sample in superscript)

p: Mutation detected and confirmed on a prior surgical specimen (also shown for each surgical sample in superscript)

No. case number, $A F$ allelic frequency, $N G S$ next generation sequencing, $d d P C R$ droplet digital PCR, $Q N S$ quantity not sufficient, $L V$ limited volume, $A D$ adequate, $A D C$ adenocarcinoma, Met Mel metastatic melanoma, Met CRC metastatic colorectal adenocarcinoma, Met PanADC metastatic pancreatic adenocarcinoma, Met UroCa metastatic urothelial carcinoma, $H C C$ hepatocellular carcinoma, Met BrADC metastatic breast adenocarcinoma, PDCa poorly differentiated carcinoma, SCC small cell carcinoma, N.A. not applicable

${ }^{\text {a} P r i o r ~ s u r g i c a l ~ s p e c i m e n ~ w a s ~ a n a l y z e d ~ u s i n g ~ a ~ d i f f e r e n t ~ N G S ~ g e n e ~ p a n e l ~ t h a t ~ d i d ~ n o t ~ e v a l u a t e ~ t h i s ~ s p e c i f i c ~ c o d o n ~ i n ~ P I K 3 C A ~(p . E 542) ~}$

${ }^{b}$ Carcinoma of unknown primary, favor cholangiocarcinoma or upper gastrointestinal

borderline cellularity (300-2000 cells per cell block section and/or 1000-5000 cells on smears) were flagged as "limited volume samples". The remaining samples that had high cellularity ( $>2000$ cells per cell block section and/or $>5000$ cells on smears) were flagged as "adequate samples".

\section{DNA extraction and mutation analysis}

DNA was extracted from $4 \mathrm{ml}$ of the supernatant samples by QIAsymphony using Qiagen circulating DNA Kit (Qiagen, Germantown, MD) as per manufacturer's guidelines. The extracted DNA was quantified using Qubit DNA high sensitivity assay kit (Thermo Fisher Scientific, Waltham, MA). Qualitative analysis of extracted supernatant DNA was performed using Agilent High Sensitivity D1000 ScreenTape on the Agilent 2200 TapeStation system (Agilent, Santa Clara, CA) according to manufacturer's instructions. In total $10 \mathrm{ng}$ supernatant DNA was used for library preparation using Ion AmpliSeq Cancer Hotspot Panel v2 and deep sequenced on Ion Proton (Thermo Fisher Scientific), as described previously [29].

Only somatic mutations were evaluated, and a minimum of 300,000 reads with a quality score of AQ20 (1 misaligned base per 100 bases) was used as a measure of successful sequencing with a minimum coverage of $\times 250$ for a wild-type call. Variants were analyzed as previously described using the Torrent Suite software v5.0.2 (Thermo Fisher Scientific) and the IT Variant Caller Plugin software v5.0.2.1 (Thermo Fisher Scientific) [5].

Specific hotspot mutations in EGFR, KRAS, BRAF, $P I K 3 C A$, and NRAS were analyzed by droplet digital PCR using available primers and probes (Bio-Rad, Hercules, CA) in a subset of the cases to confirm the mutations detected by next generation sequencing. Genotyping were performed using 10 ng DNA on QX100 Droplet Digital PCR System (Bio-Rad). PCR components were separated into individual reaction vessels using the QX100 Droplet Generator (BioRad) according to the manufacturer's instruction. Droplet reactions were subjected to PCR amplification at the following conditions: $95{ }^{\circ} \mathrm{C}$ for $10 \mathrm{~min}, 40$ cycles of $94{ }^{\circ} \mathrm{C}$ for $30 \mathrm{~s}$, and $55^{\circ} \mathrm{C}$ for $60 \mathrm{~s}$, followed by enzyme inactivation at $98{ }^{\circ} \mathrm{C}$ for $10 \mathrm{~min}$. After thermal cycling, the plates were transferred to a Droplet reader and the digital PCR data was analyzed with the QuantaSoft analytical software package (Bio-Rad).

When available, mutations detected in the supernatant fluid of the fine needle aspiration samples were compared to the results of next generation sequencing mutation analysis 
(by Ion-Torrent) performed on a prior or concurrent surgical specimen.

\section{Statistical analysis}

Statistical analysis was performed using t-test and MannWhitney test to compare results among data sets. Correlation between a pair of selected data for mutant allelic frequency in supernatant DNA was performed using Pearson correlation coefficient, and $P<0.05$ calculated by twotailed test was considered as significant.

\section{Results}

We successfully extracted DNA from supernatant fluid of fine needle aspiration samples obtained from a variety of samples, including malignant tumors $(n=25)$ and benign lymph nodes $(n=10)$ (Table 1$)$. The mean and median DNA yields from the samples was $445 \mathrm{ng}$ and $176.4 \mathrm{ng}$ (range, 15.1-2958 ng), respectively. The DNA yield from malignant tumors was significantly higher than that of the benign samples $(P<0.02)$ (Fig. 1). Qualitative analysis of the extracted supernatant DNA showed predominantly high molecular weight DNA with a small but distinct peak around 140 base pairs, suggestive of a minor component of cell-free DNA [30] admixed with DNA likely from a cellular component (Fig. 2).

Next generation sequencing was performed on the supernatant samples with a total of $88.9 \mathrm{M}$ reads obtained on Ion Proton. An average of 2,698,069 reads per sample with a quality score of AQ20 and mean sequencing depth of 5674x was achieved for the 35 samples analyzed. Somatic mutations were detected in all malignant fine needle aspiration samples tested; however no mutations were identified in any of the benign samples (Table 1).

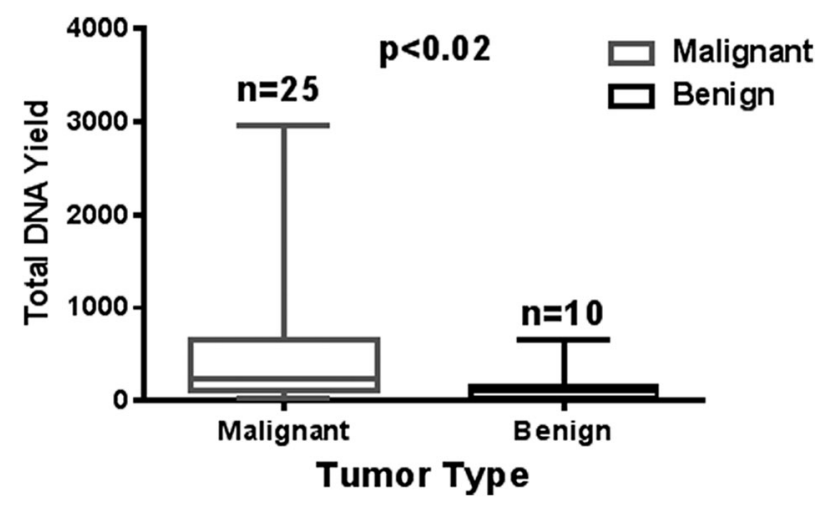

Fig. 1 Comparison of total DNA yield (ng) from supernatants of fine needle aspiration samples from malignant and benign aspirates
Two of three lung adenocarcinomas tested showed EGFR mutations and the remaining sample had a KRAS mutation. EGFR and KRAS mutations (EGFR p.L858R, EGFR p.T790M, and KRAS p.G12V) detected by next generation sequencing in the lung adenocarcinoma supernatants were confirmed by droplet digital PCR (Table 1 and Fig. 3a, b). In addition, all mutations detected in supernatant were also identified in the concurrent core needle biopsy, tested in parallel for clinical management.

Three metastatic melanoma samples showed BRAF p. V600E mutations, while the remaining 4 had NRAS mutations (p.Q61K and p.Q6IR) (Table 1). Three melanoma specimens had concurrent mutations in PIK3CA, SMAD4, or TP53 (Table 1). Similar to the lung adenocarcinomas, all $B R A F$ and NRAS mutations detected by next generation sequencing in the melanoma supernatants, were detected by droplet digital PCR, as well as identified by next generation sequencing of the concurrent core needle biopsy (cases 4, 7, and 8 ) or on a prior surgical specimen (cases 5, 6, 9, and 10).

All 4 primary pancreatic adenocarcinomas and the metastatic pancreatic adenocarcinoma to liver showed mutations in KRAS (p.G12D, p.G12V, and p.Q61H), with additional mutations in SMAD4 or TP53 detected in all five supernatants (Table 1). All KRAS mutations seen in these cases were confirmed by droplet digital PCR, including one with a low allelic frequency of $\sim 6 \%$ (case 15 ).

Of the two samples with metastatic colorectal adenocarcinoma, one had a KRAS mutation in codon 12 , confirmed by droplet digital PCR, and the other sample showed mutations in TP53 and RET (Table 1). Three samples had a diagnosis of poorly differentiated carcinoma from an unknown primary, two of which were likely from an upper gastrointestinal primary/cholangiocarcinoma, based on the clinical/radiologic findings and immunostaining profile. Supernatants from these two tumors had mutations in $I D H 1$, TP53, and PIK3CA (case 21) and in KRAS (case 23). A third unknown primary tumor supernatant showed a TP53 mutation. Both the PIK3CA and KRAS mutations were confirmed by droplet digital PCR. The remaining tumors showed a variety of somatic mutations in the supernatants as follows: metastatic urothelial carcinoma, FGFR3; metastatic breast adenocarcinoma, TP53 and PTEN; small cell carcinoma, TP53 and ATM; and hepatocellular carcinoma, TP53 (Table 1).

In addition, we evaluated the cellular component (smears, cytospin, and cell block preparations) of the malignant fine needle aspiration samples to assess the number of cases that would have qualified for next generation sequencing. Based on our criteria for molecular adequacy, 12 of the 25 malignant cases (48\%) would have qualified as "adequate samples", 7 cases (28\%) would have been rejected as "quantity not sufficient", and the remaining 
Fig. 2 Qualitative analysis of DNA extracted from supernatants of fine needle aspiration samples. The analysis shows predominantly high molecular weight DNA, likely derived out of residual cells suspended within the supernatant and/or from DNA released from cells lysed during centrifugation with a minor peak at $140 \mathrm{bp}$ (arrow) suggestive of cell-free DNA that forms a smaller component of the supernatant DNA. LM Lower Marker, UM Upper Marker

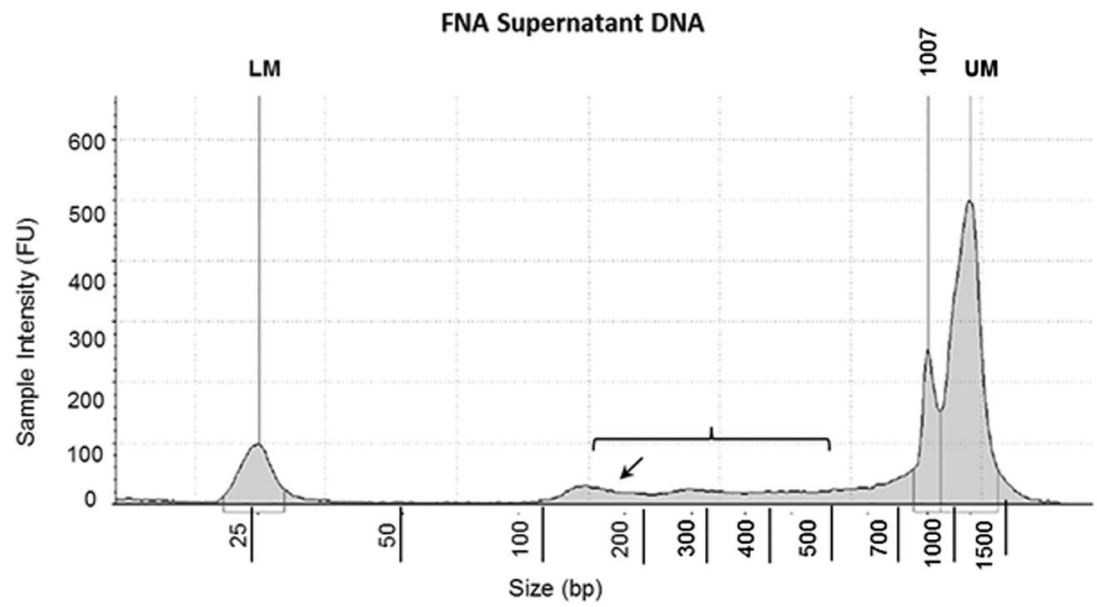

6 cases $(24 \%)$ were "limited volume samples", where either the cellular material was insufficient to perform all the clinically requested tests (next generation sequencing, fluorescence in situ hybridization, and/or IHC), or the smears could be microdissected to meet the required $20 \%$ tumor fraction, but would likely fail next generation sequencing due to low cellularity and/or inadequate DNA yields (Table 1).

\section{Discussion}

The emergence of biomarkers that play diagnostic, predictive, and prognostic roles in the clinical management of patients has moved the practice of cytopathology into the molecular arena. With an increased need for understanding the genomic landscape of solid tumors for targeted therapy, it is imperative that cytopathologists find ways of optimizing specimen handling and processing to maximize the genomic data available from small specimens [4, 31-33]. In recent years, some molecular laboratories have moved beyond the limitations of using only conventional formalin fixed paraffin embedded cell block preparations for molecular testing and have started utilizing non-formalin fixed cytologic substrates, such as direct smears and liquid based cytology. The advantages of using non-formalin fixed cytologic preparations for mutation analysis are manifold and comparative studies have demonstrated better sequencing metrics in these specimens $[2,11,34,35]$. However, given the limited tissue obtained by using an fine needle aspiration procedure, sometimes judicious triaging of the cytology sample is needed to perform the different molecular tests required for patient management [6]. Frequently this results in utilization of material on the smears, liquid based cytology, as well as the cell block preparation to meet the growing demand for molecular tests critical for patient management.
Recent studies demonstrating the feasibility of DNA extraction and molecular testing of supernatant fluid from centrifuged fine needle aspiration samples have opened the door for utilizing these samples for mutational profiling [23, 24, 27]. In addition, several institutions have reported utilizing "residual" needle rinses from fine needle aspiration samples for molecular testing [20, 36, 37]. However, a lack of awareness within the pathology community, of the utility of these substrates, likely results in an underutilization of these samples. In this study, we show that substantial amounts of DNA can be extracted and clinically relevant mutations can be detected in the otherwise discarded supernatant fluid of fine needle aspiration samples obtained from a variety of solid tumors (Fig. 4). The DNA yield from the malignant samples was significantly higher than that of the benign samples, suggesting that the malignant fine needle aspiration samples have higher numbers of tumor cells and tumor DNA shed into the aspirate, likely as a result of high cell turnover in a malignant process.

Not only were we able to demonstrate a substantial amount of DNA extracted from these samples, but we were also able to successfully perform a multiplexed next generation sequencing analysis and identify mutations relevant for the specific tumor type tested. We detected somatic mutations in all 25 malignant samples assessed in this study. None of the benign lymph nodes, included as negative controls, had any somatic mutations. In a subset of cases $(n=14)$ results from next generation sequencing on a concurrent core biopsy sample or next generation sequencing results from a prior surgical specimen were available for comparison (Table 1). All somatic mutations detected in the patient's corresponding biopsy or resection specimen were also detected in the supernatant fluids tested. It is worth emphasizing that this supernatant fluid would typically be discarded under current practice.

In addition, in a subset of cases $(n=19)$ where we had available primers and probes (corresponding to hotspots 
a

AF: $23.7 \%$; Coverage $1988 \mathrm{X}$

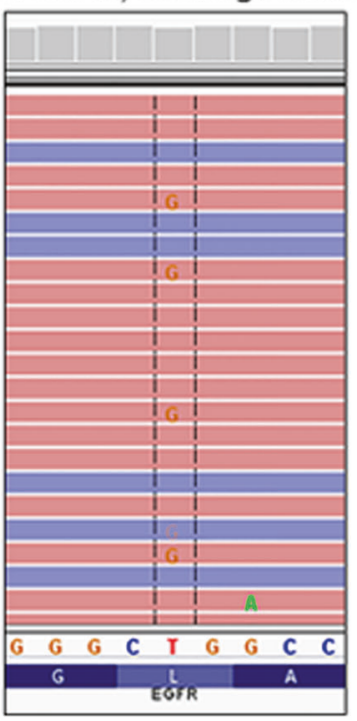

Fig. 3 Mutation analysis performed on the supernatant fluid from a lung adenocarcinoma fine needle aspiration sample (case 1). a Next generation sequencing analysis on Ion Proton using AmpliSeq Cancer Hotspot Panel v2 and visualized using Integrative Genomics Viewer (IGV), detects an EGFR mutation (c.2573T $>$ G p.L858R) at an allelic

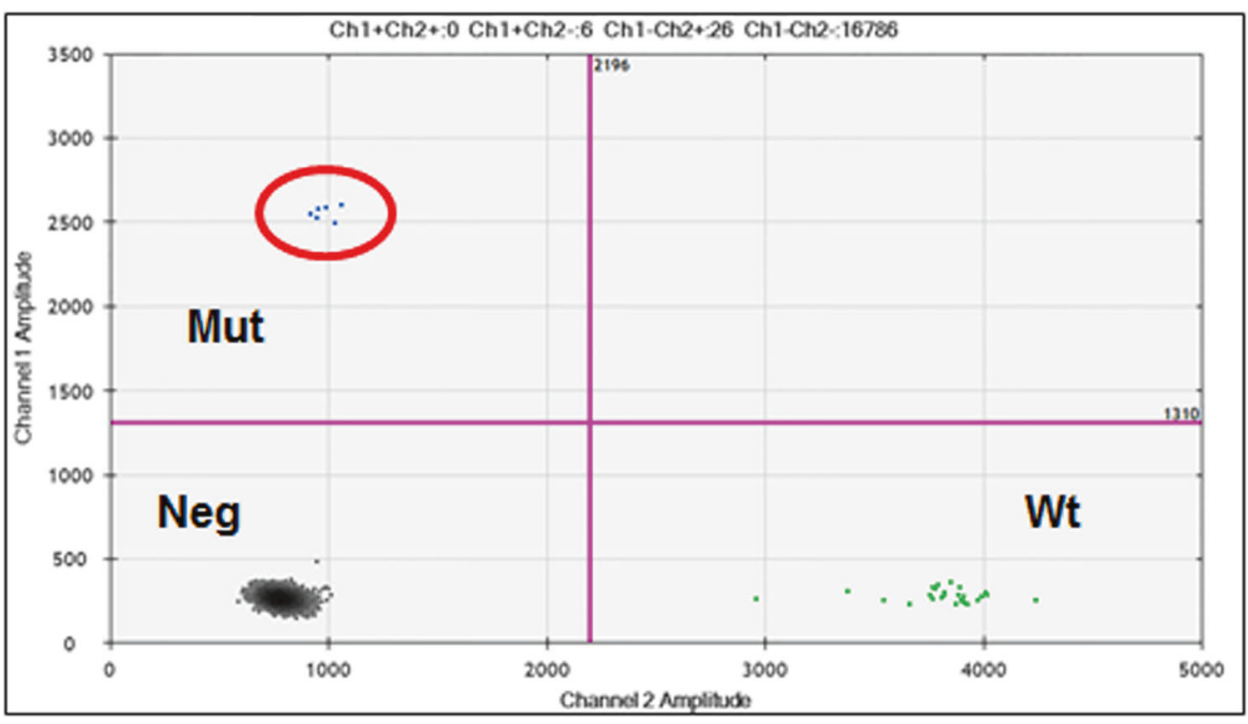

frequency of $23.7 \%$ (AF allelic frequency); b Droplet digital PCR based detection of the same EGFR c.2573T $>$ G p.L858R mutation (circle, upper left quadrant) at an allelic frequency of $19 \%$ as detected by the Droplet reader using the QuantaSoft software (Mut Mutant Droplet, Neg Negative Droplet, Wt Wild type droplet) within EGFR, KRAS, BRAF, PIK3CA, and NRAS genes), we were able to confirm these mutations by droplet digital PCR with $100 \%$ concordance $\left(R^{2}=0.9880 ; p<0.0001\right)$ (Fig. 5). The allelic frequencies observed by droplet digital PCR corresponded well with those seen by next generation sequencing (Table 1), thus serving as a confirmatory assay on an orthogonal/ alternate platform.

In this study we also show distinct advantages of being able to detect the mutations using droplet digital PCR. These advantages include, (1) improved turnaround time of 1 day post-extraction vs. 5 days for next generation sequencing; (2) cost effective, as a single droplet digital PCR assay can be performed with an approximate cost of $\$ 9.5 /$ sample vs. $\$ 400 /$ sample for a 50 -gene next generation sequencing assay; (3) high analytic sensitivity of $\sim 0.1 \%$ vs. 5-10\% for next generation sequencing, which allows for an ultrasensitive method for detecting mutations from low tumor fraction samples. The latter may be of relevance in post-treatment cases where obtaining an adequate tumor fraction on a fine needle aspiration sample may be difficult due to extensive fibrosis. Therefore, in cases where the clinical question is specific, for example identifying EGFR p.T790M resistance mutation in a lung adenocarcinoma patient or BRAF p.V600E mutation in a melanoma or thyroid carcinoma patient, performing droplet digital PCR from a supernatant sample may offer the advantage of a rapid, sensitive, and relatively inexpensive assay. On the other hand, droplet digital PCR is designed for specific hotspot mutations and does not provide the multiplexed advantage of next generation sequencing for interrogating multiple genes using small amounts of input DNA.

A subset of the mutations detected in our next generation sequencing assay had predictive and prognostic significance. For example, the detection of the EGFR resistance mutation p.T790M in a lung adenocarcinoma patient (case 2) treated with tyrosine kinase inhibitors (TKI) implies resistance to therapy and would prompt the clinician to switch to third-generation TKI therapy. Patients with BRAF p.V600E mutations detected (cases 4, 8, and 9) would be eligible for BRAF inhibitor therapy, whereas patients with EGFR sensitizing mutation p.L858R (case 1) would qualify for targeted TKI therapy. While the detection of certain mutations may have prognostic implications such as KRAS mutations in pancreatic adenocarcinoma (cases 12, 13, 14, and 15) and FGFR3 mutation in urothelial carcinoma (case 17), other mutations detected may have diagnostic significance. For example, case 21 was a poorly differentiated carcinoma of unknown primary where the immunostaining profile of the tumor was suggestive of a cholangiocarcinoma or upper gastrointestinal primary neoplasm; next generation sequencing detected an IDH1 mutation (TP53 and PIK3CA mutations were also present), a mutation seen in 11-24\% of intrahepatic cholangiocarcinomas [38, 39], which would lend support to the suspected diagnosis. 


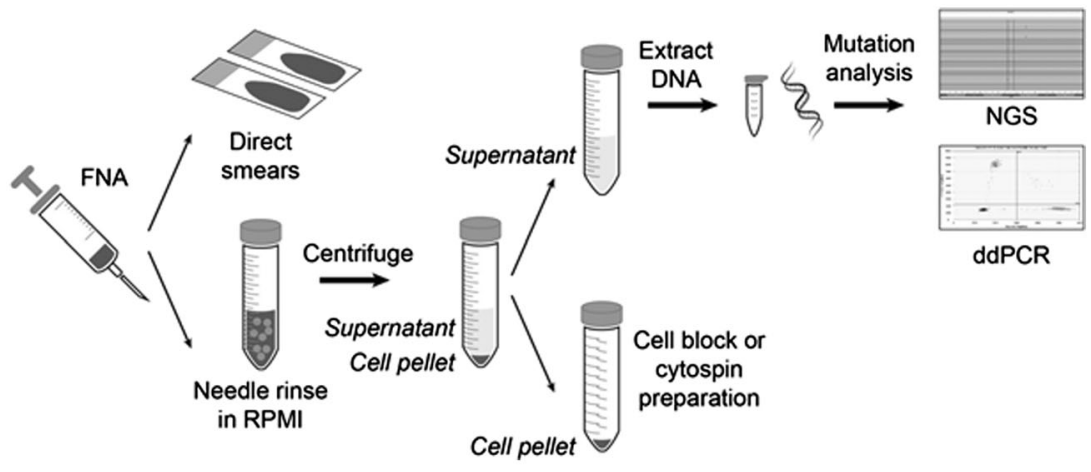

Fig. 4 Schematic diagram showing the workflow of mutational profiling of supernatant fluids obtained from centrifugation of fine needle aspiration samples. Direct smears are prepared for staining and diagnosis, while the needle rinse is collected in RPMI and subsequently centrifuged to create a cell pellet for either cell block or cytospin preparation. The supernatant fluid from the centrifuged sample is collected for DNA extraction and subsequent mutation analysis by next generation sequencing and/or droplet digital PCR

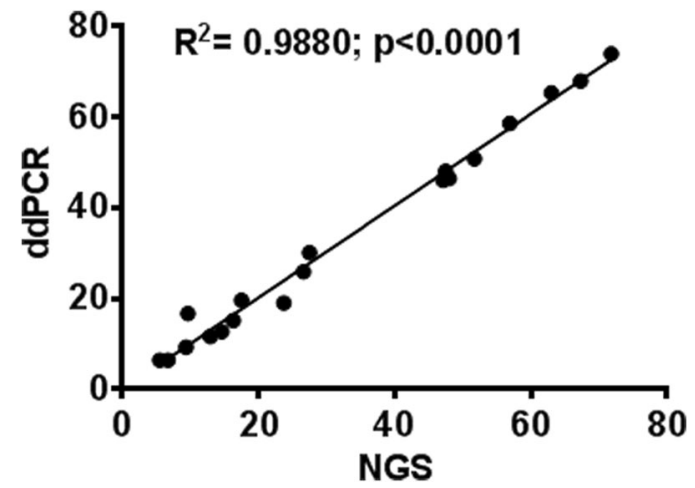

Fig. 5 Comparing mutation detection by next generation sequencing platform (Ion Proton) and droplet digital PCR in DNA extracted from fine needle aspiration supernatant samples shows high concordance $\left(R^{2}=0.0988 ; p<0.0001\right)$

The ability to detect somatic mutations from otherwise discarded supernatant fluids offers the ability to triage and effectively utilize limited volume fine needle aspiration samples when multiple molecular tests are requested. For example, at our institution lung adenocarcinomas typically have the following tests requested: fluorescence in situ hybridization assays (ALK, ROS1, RET and MET), immunocytochemistry (PD-L1, BRAF, MET), and mutation analysis by next generation sequencing (EGFR, KRAS, and $B R A F$ ). In cases where the cellular material (smears, liquid based cytology, and/or cell block preparation) is insufficient to perform all the requested tests, the supernatant can be utilized for mutation testing, while the cellular material can be used for fluorescence in situ hybridization and immunocytochemistry. Our data shows that supernatants from limited volume fine needle aspiration samples and samples that were deemed "quantity not sufficient" (13 of 25 (52\%) cases, based on review of the corresponding fine needle aspiration smears, cytospins and/or cell block sections)

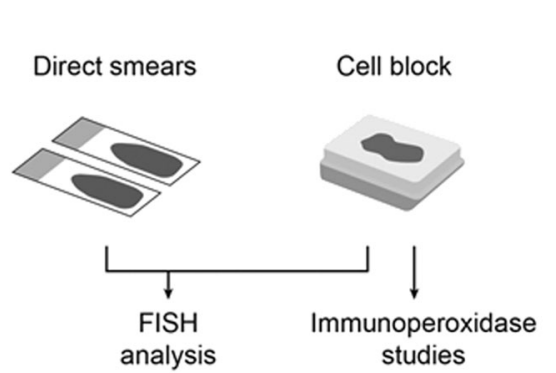

Supernatant from needle rinse

Fig. 6 Proposed workflow algorithm to triage cases with multiple biomarker requests on limited tissue, for effective and judicious use of fine needle aspiration samples

yielded substantial amounts of DNA and were able to detect clinically relevant somatic mutations. Thus, mutational analysis of supernatants would be extremely valuable, especially in cases with limited tissue, allowing more efficient utilization of patient samples and preventing a rebiopsy for additional tissue samples. A proposed workflow algorithm in such cases is shown in Fig. 6.

Mutation allelic frequencies seen in the next generation sequencing analysis showed a wide range (5.5-90.7\%), which likely reflects the relative amount of tumor DNA in the samples. Although it is difficult to estimate tumor fraction in a supernatant sample, a low allelic frequency such as the one seen in case $15(K R A S, 5.5 \%)$ would indicate an overall low tumor fraction in the cytology specimen. Indeed, a review of the corresponding cytology smears showed rare fragments of adenocarcinoma in a background of predominantly benign epithelial cells. Therefore, if the mutation allelic frequency corresponds to the amount of tumor cells seen in the fine needle aspiration sample, this data lends some evidence to the hypothesis that the tumor DNA detected in these samples is either derived from cells that remain suspended in the supernatant post- 
centrifugation or DNA from cells that are disrupted in the centrifugation process releasing DNA into the supernatant fluid. This is further supported by the qualitative analysis of the supernatant DNA that clearly indicated a predominant high molecular weight DNA component (likely from a cellular component) admixed with a small amount of cellfree DNA [30] (Fig. 1). While, the detection of a somatic mutation from the supernatant fluid of a malignant fine needle aspiration sample is helpful for patient care, a negative result needs to be interpreted with some caution, since the assessment of tumor fraction in these samples would be difficult. Therefore, further studies using a larger cohort (including both positive and negative cases) and controlled pre- analytic factors will be needed for a thorough qualitative analysis of the supernatant DNA and determination of the sensitivity and lowest limit of detection (of mutations) from supernatant samples.

None of the 10 benign lymph node samples that were selected as a negative control in this study demonstrated any mutation detected by next generation sequencing. However, in the event of a somatic mutation detected in the supernatant fluid from a cytologically benign aspirate, one would need to exclude a false positive result and confirm the mutation by (1) repeating the assay to check for reproducibility of detected mutation; (2) confirm the mutation detected by evaluating on an orthogonal platform, if available; (3) re-review the cytology slides to confirm the cytologic diagnosis; (4) repeat the assay on the cytologic cellular material (smears/cell block) to see if the mutation detected in the supernatant is present in the fine needle aspiration cellular material; and (5) exclude the possibility of a germline variant [40, 41]. With ultrasensitive mutational assays such as droplet digital PCR, detecting a somatic mutation from circulating or cell-free tumor DNA is also a remote possibility and therefore evaluating peripheral blood or plasma from the patient may help in the determination.

In summary, our study has successfully shown the utility and potential value of centrifuged supernatant fluid from fine needle aspiration needle rinses, which are typically discarded. Our results show that, these supernatants provide a robust substrate for expanded mutation profiling by next generation sequencing, as well as hotspot mutation testing by droplet digital PCR. Testing the fine needle aspiration supernatant will facilitate mutation testing in patients where the cytology sample contains limited amounts of tumor, without the need to re-biopsy for additional tissue samples.

Acknowledgements This work was supported in part by The University of Texas MD Anderson Cancer Center Shirley Stein Scientific Endowed Research Award. We want to thank Kim-Anh Vu for technical assistance with graphics design.

\section{Compilance with ethical standards}

Conflict of interest The authors declare that they have no conflict of interest.

\section{References}

1. Aisner DL, Rumery MD, Merrick DT, et al. Do more with less: tips and techniques for maximizing small biopsy and cytology specimens for molecular and ancillary testing: the university of colorado experience. Arch Pathol Lab Med. 2016;140:1206-1220.

2. Roy-Chowdhuri S, Chen H, Singh RR, et al. Concurrent fine needle aspirations and core needle biopsies: a comparative study of substrates for next-generation sequencing in solid organ malignancies. Mod Pathol. 2017;30:499-508.

3. Coley SM, Crapanzano JP, Saqi A. FNA, core biopsy, or both for the diagnosis of lung carcinoma: Obtaining sufficient tissue for a specific diagnosis and molecular testing. Cancer Cytopathol. 2015;123:318-26.

4. VanderLaan PA. Molecular markers: Implications for cytopathology and specimen collection. Cancer Cytopathol. 2015;123:454-60.

5. Roy-Chowdhuri S, Goswami RS, Chen H, et al. Factors affecting the success of next-generation sequencing in cytology specimens. Cancer Cytopathol. 2015;123:659-68.

6. Roy-Chowdhuri S, Stewart J. Preanalytic variables in cytology: lessons learned from next-generation sequencing-the MD anderson experience. Arch Pathol Lab Med. 2016;140:1191-1199.

7. Goswami RS, Luthra R, Singh RR, et al. Identification of factors affecting the success of next-generation sequencing testing in solid tumors. Am J Clin Pathol. 2016;145:222-37.

8. Kanagal-Shamanna R, Portier BP, Singh RR, et al. Nextgeneration sequencing-based multi-gene mutation profiling of solid tumors using fine needle aspiration samples: promises and challenges for routine clinical diagnostics. Mod Pathol. 2014;27:314-27.

9. Chen H, Luthra R, Goswami RS, Singh RR, Roy-Chowdhuri S. Analysis of pre-analytic factors affecting the success of clinical next-generation sequencing of solid organ malignancies. Cancers. 2015;7:1699-715.

10. Gleeson FC, Kipp BR, Kerr SE, et al. Characterization of endoscopic ultrasound fine-needle aspiration cytology by targeted nextgeneration sequencing and theranostic potential. Clin Gastroenterol Hepatol. 2015;13:37-41.

11. Karnes HE, Duncavage EJ, Bernadt CT. Targeted next-generation sequencing using fine-needle aspirates from adenocarcinomas of the lung. Cancer Cytopathol. 2014;122:104-13.

12. Nikiforov YE, Carty SE, Chiosea SI, et al. Highly accurate diagnosis of cancer in thyroid nodules with follicular neoplasm/ suspicious for a follicular neoplasm cytology by ThyroSeq v2 next-generation sequencing assay. Cancer. 2014;120:3627-34.

13. Nikiforova MN, Wald AI, Roy S, Durso MB, Nikiforov YE. Targeted next-generation sequencing panel (ThyroSeq) for detection of mutations in thyroid cancer. J Clin Endocrinol Metab. 2013;98:E1852-1860.

14. Scarpa A, Sikora K, Fassan M, et al. Molecular typing of lung adenocarcinoma on cytological samples using a multigene next generation sequencing panel. PLoS ONE. 2013;8:e80478.

15. Wei S, LiVolsi VA, Montone KT, Morrissette JJ, Baloch ZW. Detection of molecular alterations in medullary thyroid carcinoma using next-generation sequencing: an institutional experience. Endocr Pathol. 2016;27:359-62. 
16. Young G, Wang K, He J, et al. Clinical next-generation sequencing successfully applied to fine-needle aspirations of pulmonary and pancreatic neoplasms. Cancer Cytopathol. 2013;121:688-94.

17. Reynolds JP, Zhou Y, Jakubowski MA, et al. Next-generation sequencing of liquid-based cytology non-small cell lung cancer samples. Cancer. 2017;125:178-87.

18. Baum JE, Zhang P, Hoda RS, et al. Accuracy of next-generation sequencing for the identification of clinically relevant variants in cytology smears in lung adenocarcinoma. Cancer. 2017; 125:398-406.

19. Treece AL, Montgomery ND, Patel NM, et al. FNA smears as a potential source of DNA for targeted next-generation sequencing of lung adenocarcinomas. Cancer Cytopathol. 2016;124:406-14.

20. Fuller MY, Mody D, Hull A, Pepper K, Hendrickson H, Olsen R. Next-generation sequencing identifies gene mutations that are predictive of malignancy in residual needle rinses collected from fine-needle aspirations of thyroid nodules. Arch Pathol Lab Med. 2017.

21. Velizheva NP, Rechsteiner MP, Wong CE, et al. Cytology smears as excellent starting material for next-generation sequencingbased molecular testing of patients with adenocarcinoma of the lung. Cancer. 2017;125:30-40.

22. Bellevicine C, Sgariglia R, Malapelle U, et al. Young investigator challenge: can the ion AmpliSeq cancer hotspot panel v2 be used for next-generation sequencing of thyroid FNA samples? Cancer. 2016;124:776-84.

23. Deftereos G, Finkelstein SD, Jackson SA, et al. The value of mutational profiling of the cytocentrifugation supernatant fluid from fine-needle aspiration of pancreatic solid mass lesions. Mod Pathol. 2014;27:594-601.

24. Finkelstein SD, Bibbo M, Kowalski TE, et al. Mutational analysis of cytocentrifugation supernatant fluid from pancreatic solid mass lesions. Diagn Cytopathol. 2014;42:719-25.

25. Finkelstein SD, Bibbo M, Loren DE, et al. Molecular analysis of centrifugation supernatant fluid from pancreaticobiliary duct samples can improve cancer detection. Acta Cytol. 2012;56:439-47.

26. Malhotra N, Jackson SA, Freed LL, et al. The added value of using mutational profiling in addition to cytology in diagnosing aggressive pancreaticobiliary disease: review of clinical cases at a single center. BMC Gastroenterol. 2014;14:135.

27. Brown AE, Lim KS, Corpus G, Hustek MT, Tran TA, Chang CC. Detection of BRAF mutation in the cytocentrifugation supernatant fluid from fine-needle aspiration of thyroid lesions may enhance the diagnostic yield. Cytojournal. 2017;14:4.
28. Billah S, Stewart J, Staerkel G, Chen S, Gong Y, Guo M. EGFR and KRAS mutations in lung carcinoma: molecular testing by using cytology specimens. Cancer Cytopathol. 2011;119:111-7.

29. Mehrotra M, Singh RR, Chen W, et al. Study of preanalytic and analytic variables for clinical next-generation sequencing of circulating cell-free nucleic acid. J Mol Diagn. 2017;19:514-24.

30. Underhill HR, Kitzman JO, Hellwig S, et al. Fragment length of circulating tumor DNA. PLoS Genet. 2016;12:e1006162.

31. Vigliar E, Malapelle U, de Luca C, Bellevicine C, Troncone G. Challenges and opportunities of next-generation sequencing: a cytopathologist's perspective. Cytopathology. 2015;26:271-83.

32. Rekhtman N, Roy-Chowdhuri S. Cytology specimens: a goldmine for molecular testing. Arch Pathol Lab Med. 2016;140:1189-90.

33. Roh MH. The utilization of cytologic fine-needle aspirates of lung cancer for molecular diagnostic testing. J Pathol Transl Med. 2015;49:300-9.

34. Gailey MP, Stence AA, Jensen CS, Ma D. Multiplatform comparison of molecular oncology tests performed on cytology specimens and formalin-fixed, paraffin-embedded tissue. Cancer Cytopathol. 2015;123:30-9.

35. Hwang DH, Garcia EP, Ducar MD, Cibas ES, Sholl LM. Nextgeneration sequencing of cytologic preparations: An analysis of quality metrics. Cancer. 2017;125:786-94.

36. Wei S, Lieberman D, Morrissette JJ, Baloch ZW, Roth DB, McGrath C. Using "residual" FNA rinse and body fluid specimens for next-generation sequencing: an institutional experience. Cancer Cytopathol. 2016;124:324-9.

37. Tian SK, Killian JK, Rekhtman N, et al. Optimizing workflows and processing of cytologic samples for comprehensive analysis by next-generation sequencing: memorial sloan kettering cancer center experience. Arch Pathol Lab Med. 2016;140:1200-1205.

38. Borger DR, Tanabe KK, Fan KC, et al. Frequent mutation of isocitrate dehydrogenase (IDH) 1 and IDH2 in cholangiocarcinoma identified through broad-based tumor genotyping. Oncologist. 2012;17:72-9.

39. Kipp BR, Voss JS, Kerr SE, et al. Isocitrate dehydrogenase 1 and 2 mutations in cholangiocarcinoma. Hum Pathol. 2012;43:1552-8.

40. Hu Y, Alden RS, Odegaard JI, et al. Discrimination of germline EGFR T790M mutations in plasma cell-free DNA allows study of prevalence across 31,414 cancer patients. Clin Cancer Res. 2017;23:7351-9.

41. Sukari A, Nagasaka M, Wakeling E. EGFR-mutant non-small cell lung cancer in the era of precision medicine: importance of germline EGFR T790M testing. J Natl Compr Canc Netw. 2017;15:1188-92. 